\title{
Tunneling spectra of break junctions involving $\mathrm{Nb}_{3} \mathrm{Sn}$
}

\author{
Toshikazu Ekino, Akira Sugimoto, and Yuta Sakai \\ Hiroshima University, Graduate school of Integrated Arts and Sciences, Higashihiroshima 739-8521, Japan \\ E-mail: ekino@hiroshima-u.ac.jp
}

Alexander M. Gabovich

Institute of Physics, National Academy of Sciences of Ukraine, Kiev 03680, Ukraine

Jun Akimitsu

Department of Physics, Aoyama-Gakuin University, Sagamihara 252-5277, Japan

Received June 2, 2014, published online August 21, 2014

\begin{abstract}
The electronic gap structure of $\mathrm{Nb}_{3} \mathrm{Sn}$ was measured by the break-junction (BJ) tunneling technique. The superconducting gap values are estimated to be in the range $2 \Delta=4-5.5 \mathrm{meV}$ at $T=4.2 \mathrm{~K}$ as follows from the observed distinct conductance peaks. In addition to the superconducting gap structure, we observed reproducible hump-like structures at the biases of about \pm 20 and $\pm 50 \mathrm{mV}$. Such a coexistence of gap and hump structures resembles the situation found in the high- $T_{c}$ copper-oxide superconductors. Above the superconducting critical temperature $T_{C} \sim 18 \mathrm{~K}$, the humps appear as the only gap-like structures. Their possible origin is discussed in connection to the structural phase transition occurring in $\mathrm{Nb}_{3} \mathrm{Sn}$.
\end{abstract}

PACS: 74.55.+v Tunneling phenomena: single particle tunneling and STM;

74.70.Ad Metals; alloys and binary compounds;

71.20.-b Electron density of states and band structure of crystalline solids;

74.81.Fa Josephson junction arrays and wire networks;

81.30.Kf Martensitic transformations.

Keywords: tunneling, break junction, superconducting energy gap, $\mathrm{Nb}_{3} \mathrm{Sn}$, charge density waves.

\section{Introduction}

Among the inter-metallic superconductors with A-15 cubic crystal structure, $\mathrm{Nb}_{3} \mathrm{Sn}$ is one of the most popular compounds because of its relatively high $T_{C}=18 \mathrm{~K}$ and stable metallurgical characteristics [1]. Its crystal structure is not the layered one in contrast to the copper-oxide high $T$ superconductors, but instead $\mathrm{Nb}$ atoms form orthogonal linear chains along each principal cubic direction [2]. Such $\mathrm{Nb}$ atomic chains were considered to be related to high- $T$ superconductivity of the A-15 structure. It is well known that the A-15 cubic compounds undergo the tetragonal distortion due to the electronic instability of the linear chains of transition metal atoms (like $\mathrm{Nb}$ ) at the characteristic temperature $T_{m}$, which is higher than $T_{c}$. This phase transition is a martensitic one, and the antagonistic interplay between structural instability and superconductivity has been investigated both experimentally and theoretically $[1,3,4]$. To elucidate the character of superconductivity in
A-15 compounds, tunnel junctions and electron tunneling spectroscopy were intensively applied to measure the superconducting gap $2 \Delta$ and the electron-phonon interaction described by the Eliashberg function $\alpha^{2} F(\omega)$ [5-7]. Almost all the junctions fabricated so far in order to perform spectroscopic measurements involved artificial oxide barriers, which might lead to the emergence of spurious features in the tunneling spectra. Break junctions of Nb-Sn filaments [8] was the only exception.

In this paper we present tunneling measurements of $\mathrm{Nb}_{3} \mathrm{Sn}$ single crystals using up-graded break-junction (BJ) technique. The studies were focused on the superconducting gap characteristics as well as the electronic features related to the martensitic transition. The state below $T_{m}$ is believed to be one with charge-density waves (CDWs) induced by the Peierls instability [3]. The electronic gap formation in the density of states is one of the main consequences of the CDW transition, which can be exactly de- 
tected by the BJ technique. Actually, recent point-contact spectroscopic studies of $\mathrm{Nb}_{3} \mathrm{Sn}$ not involving artificial barriers confirmed such a viewpoint [9].

\section{Experimental procedures}

The single crystals were grown by the vapor transport method. To characterize the sample, the temperature, $T$, dependence of the electrical resistance, $R(T)$, is shown in the inset of Fig. 1. The BJ technique was used to form the appropriate junction interface. The crucial advantage of this technique is a cryogenic fracture at $4.2 \mathrm{~K}$ of the sample fixed on the flexible substrate with four electrodes by applying an external bending force, resulting in a fresh and clean junction interface that can provide the unaffected gap features [10]. This junction design forms a superconductor-insulator-superconductor (SIS) symmetric junction structure.

\section{Results and discussion}

Figure 1 shows the tunneling conductance $G(V)=$ $=d I / d V(V)$ at $4.2 \mathrm{~K}$ for different BJs. A sharp and intensive gap-edge structure is inherent to the bottom curve showing the peak-to-peak voltage interval $V_{p-p} \approx 8.6 \mathrm{mV}$ (corresponding biases are $\pm 4.3 \mathrm{mV}$ ) and $\sim 10 \%$ zero-bias leakage as compared to the value $G( \pm 20 \mathrm{mV})$. This is typical of the Bardeen-Cooper-Schrieffer (BCS) gap structure with the SIS junction geometry of BJ, thereby $V_{p-p}=4 \Delta / e$. Here $e$ is the elementary charge. The sub-gap peaks at $\pm 2.3 \mathrm{mV}$ appear due to the $\pm \Delta$ /e singularities as a result of the partial formation of SIN ( $\mathrm{N}$ stands for a normal metal) junctions. The gap value $2 \Delta=4.6 \mathrm{meV}$ in fact corresponds to the Sn deficient region in the crystal [6].

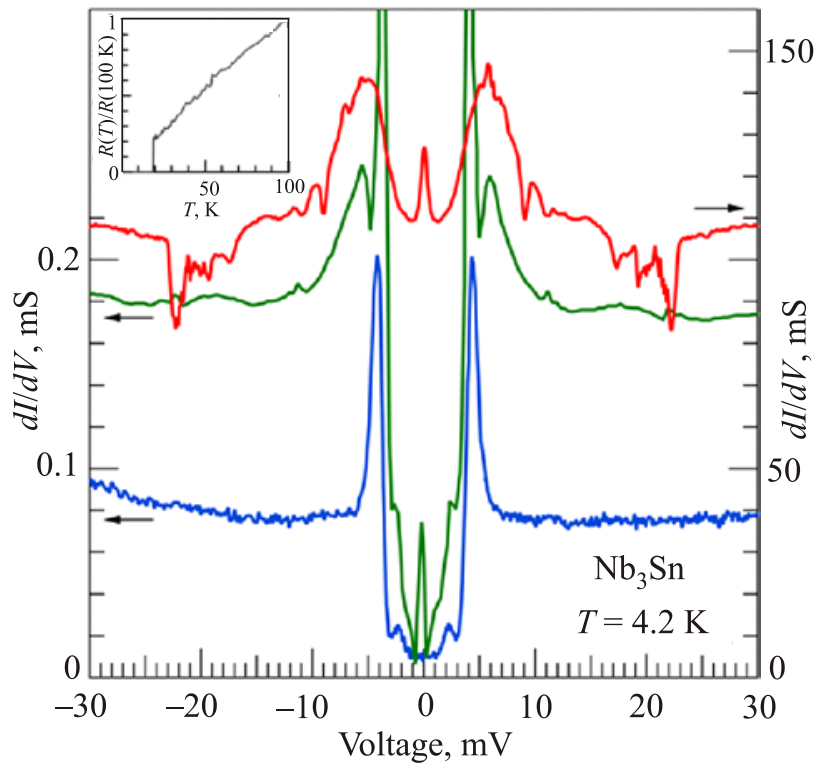

Fig. 1. (Color online) Tunneling conductance $G(V)=d I / d V(V)$ obtained for different $\mathrm{Nb}_{3} \mathrm{Sn}$ break junctions in the superconducting state at $4.2 \mathrm{~K}$. The inset shows the temperature dependence of the electrical resistance $R(T)$ normalized by $R(T=100 \mathrm{~K})$.
In contrast, the gap peak in $G(V)$ for the top curve is conspicuously broadened showing quite high conductance leakage as well as the factor of $\sim 10^{3}$ larger high-bias conductance magnitude. The conductance leakage almost reaches the normal-state high-bias value and also demonstrates the Josephson (weak-link) zero-bias peak. There is a substantial quasiparticle scattering at the interface involving the Andreev reflection. The broad conductance peaks at $\pm 5.5 \mathrm{mV}$ corresponding to $\pm 2 \Delta / e$ of an SIS junction are larger than $\pm 4.3 \mathrm{mV}$ of the bottom curve. In the middle curve, the observed features include both gap values appropriate to the junctions described above. The rather broad $\pm 5.5 \mathrm{mV}$ peaks outside the $\pm 4.2 \mathrm{mV}$ peaks are consistent with those of the top curve, thereby indicating the inherent gap of $\mathrm{Nb}_{3} \mathrm{Sn}$, although the appearance of the gap is not as intensive as in the top curve. On the other hand, the $\pm 5.5 \mathrm{mV}$ peaks are absent when the zero-bias peak is suppressed, as is shown in the bottom curve. Since the zero-bias peak corresponds to the coherent Cooper-pair tunneling process and may not manifest itself in the disordered regions, the absence of the feature supports the idea that the BJ interface is formed along the immature superconducting phase in the nonideal Nb-Sn composite region in the crystal.

Assuming the isotropic BCS gap to $T_{C}$ ratio $2 \Delta / k_{B} T_{C} \approx$ $\approx 3.52$, where $k_{B}$ is the Boltzmann constant, the gap values inferred from the experiment correspond to the local critical temperatures $T_{c}^{*}=14 \mathrm{~K}$ (bottom curve) $-18 \mathrm{~K}$ (top curve). The former value corresponds to the gap of a nonstoichiometric phase in spite of the observed sharp gap edge, while the latter value is the bulk $T_{\mathcal{C}}$, although $G(V)$ is substantially distorted in this case. Anyway, the strong-coupling gap value of $2 \Delta>6.5-7 \mathrm{meV}$, which can be deduced from the widely accepted gap ratio $2 \Delta / k_{B} T_{C}=4.3-4.4$ and the bulk $T_{C}=18 \mathrm{~K}$ [6], was not found in our measurements.

In Fig. 2 we demonstrate the temperature evolution of $G(V)$ obtained for a mechanically stable BJ. The shape of $G(V)$ at each $T$ is smeared but is quite stable in the whole $T$ range from $4.2 \mathrm{~K}$ up to $\sim 17.4 \mathrm{~K}$. The gap-edge peaks at $6.4 \mathrm{~K}$, which occur at the same biases as in Fig. 1, are gradually suppressed and smeared with increasing $T$. The observed behavior is typical for BCS superconductors. The inset shows the SIS conductance fitting results using the Dynes equation with thermal smearing in order to determine accurately the gap value at $6.4 \mathrm{~K} \mathrm{[11].} \mathrm{We} \mathrm{can} \mathrm{recognize}$ from these results that the fitted gap-peak position corresponding to the gap parameter $\Delta=2-2.2 \mathrm{meV}$ does not change substantially even if the phenomenological broadening parameter $\Gamma$ is drastically varied $(\Gamma=0.12-0.46 \mathrm{meV})$. From the $T$ evolution of the gap characteristics, we determine the critical temperature $T_{C}$ at the $\mathrm{BJ}$ interface as 17.5 $\mathrm{K}$, which is close to the bulk $T_{C}=18 \mathrm{~K}$. The low- $T$ gap combined with the value $T_{C} \approx 17.5 \mathrm{~K}$ yields the gap to $T_{C}$ ratio $2 \Delta / k_{B} T_{C} \approx 2.7-3$, which is far from the literature strong-coupling value 4.4 [6]. Close look at the curve set in 


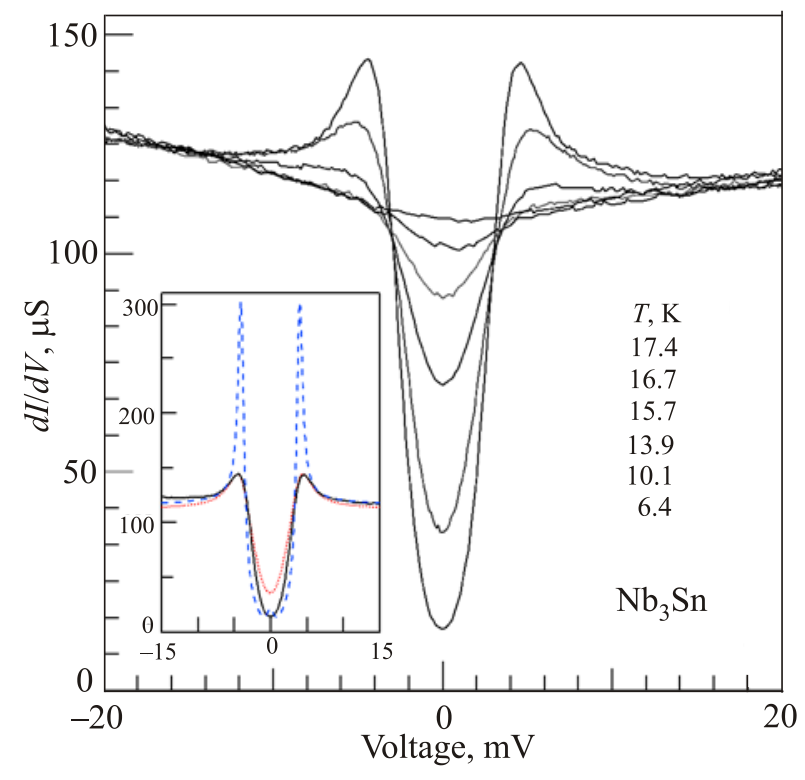

Fig. 2. (Color online) Temperature variation of $G(V)$ raw data for a $\mathrm{Nb}_{3} \mathrm{Sn}$ break junction. The inset shows the SIS fitting results for the $6.4 \mathrm{~K}$ curve using the Dynes equation with thermal smearing [11]. The dashed and dotted curves correspond to the broadening parameters $\Gamma=0.12 \mathrm{meV}$ and $0.46 \mathrm{meV}$, respectively, for the same gap parameter $\Delta=2 \mathrm{meV}$.

Fig. 2 shows that the gap structure develops below 14-16 K, which is consistent with the local BCS value $T_{c}^{*} \approx 14 \mathrm{~K}$ corresponding to $2 \Delta=4.2 \mathrm{meV}$ as was evaluated from data demonstrated in Figs. 1 and 2. This means that the small gap is induced by the proximity effect at the junction region presumably due to the BJ fracture of the local superconducting phase in the crystal.

Looking at larger-bias region of Fig. 1 more carefully, one can readily see the broad humps around $\pm 20 \mathrm{mV}$ being a third structure in the middle curve along with the double-gap features at $V \approx \pm 4.3 \mathrm{mV}$ and $\pm 5.5 \mathrm{mV}$. Humps at $\pm 20 \mathrm{mV}$ are similar for different junctions. Namely, the bias positions and the humps magnitudes of about 2-4\% excess above the background are almost the same. Such conductance structures at $\pm 20 \mathrm{mV}$ found here have been found previously in the $\mathrm{Nb}-\mathrm{Sn} \mathrm{BJ}$ [8]. A rather small amplitude of $2-4 \%$ testifies that structures at $\pm 20 \mathrm{mV}$ might be associated with the strong-coupling effect of the phonons, since the electron-phonon mechanism of superconductivity is expected to dominate in $\mathrm{Nb}_{3} \mathrm{Sn}$. However, this conclusion seems not to be valid in this case, because similar hump-like structures are also observed at $\pm 4.3 \mathrm{mV}$ in the absence of the $\pm 5.5 \mathrm{mV}$ gap structures, inherent to the full-developed superconducting state. Furthermore, although the strong-coupling effects of the electron-phonon interaction should be seen in every $G(V)$ with the ideal BCS-like gap structures, no such feature was found in $G(V)$, which demonstrated very distinct gap structure (Fig. 1, bottom curve).
At the same time, a coexistence in the conductance spectra of the superconducting and smeared hump structures resembles the features found in tunneling conductance of the high- $T$ cupper-oxide superconductors at low temperatures [12]. The similarity between the hump feature at $\pm 20 \mathrm{mV}$ and the normal-state gap manifestation in the high- $T$ superconductor can be inferred from Fig. 3, where the $G(V)$ from different BJs are presented at temperatures up to well above $T_{C}$, if one bears in mind the difference in energy scales. The $G(V)$ shapes displayed in Fig. 3 shows that the asymmetry of the gap-edge peaks with respect to the zero-bias varies for various junctions, but the peak locations at \pm (20-30) $\mathrm{mV}$ are approximately the same.

From the facts discussed above, the large-bias peculiarities observed here as well as those reported previously for $\mathrm{Nb}_{3} \mathrm{Sn}[5-8,13,14]$, which were attributed to the electronphonon-interaction manifestations, should rather be considered as the gap-edge structures of a nonsuperconducting nature. Moreover, the $\pm(20-30) \mathrm{mV}$ structures in $\mathrm{Nb}_{3} \mathrm{Sn}$ survive regularly at temperatures well above $T_{C}$ similarly to the transformation of the large-bias tunnel conductance features into the pseudogap depression in the normal state observed in cuprates $[14,15]$. We want to emphasize that in the $23.9 \mathrm{~K}$ curve of Fig. 3 the fairly well defined peaks of $G(V)$ occur at biases at $\approx \pm 40 \mathrm{mV}$ in addition to the inner $-20 \mathrm{mV}$ peak or $+20 \mathrm{mV}$ hump structures. The outer value is twice as much as the inner one.

In Fig. 4 the conductance $G(V)$ is displayed for different BJs in the large bias range $|V| \leq 120 \mathrm{mV}$ at $T=4.2 \mathrm{~K}$ and higher $T>T_{C}$. The $G(V)$ in the superconducting state at $4.2 \mathrm{~K}$ demonstrates the coherent superconducting gap-edge peaks at $\pm 4.5 \mathrm{mV}$, which correspond to $\pm 2 \Delta / e$, the Josephson peak

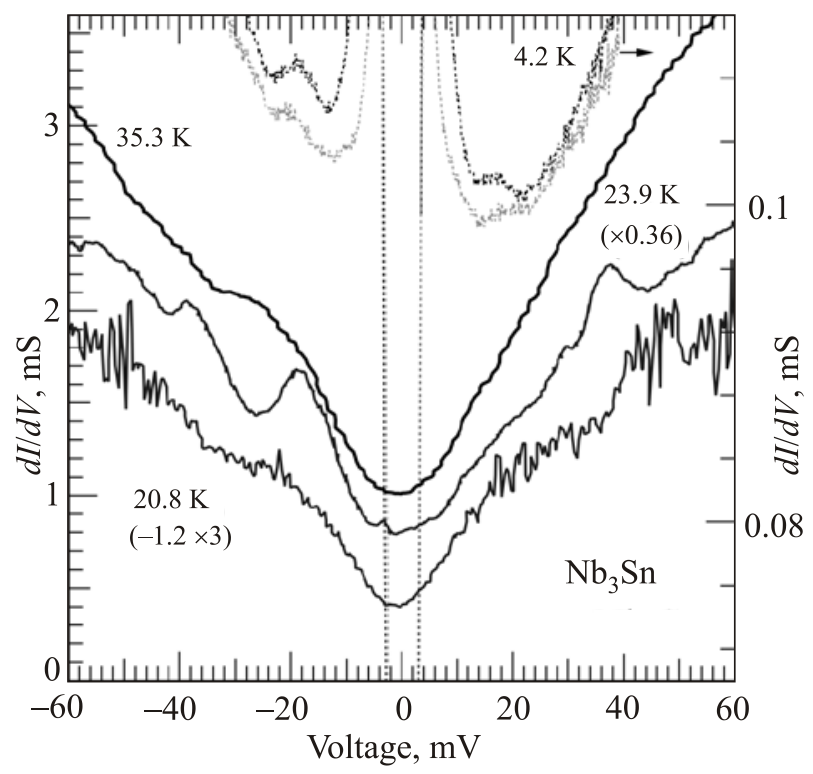

Fig. 3. $G(V)$ for different $\mathrm{Nb}_{3} \mathrm{Sn}$ break junctions in the superconducting and normal states: comparison of the $\pm 20 \mathrm{mV}$ structures. 


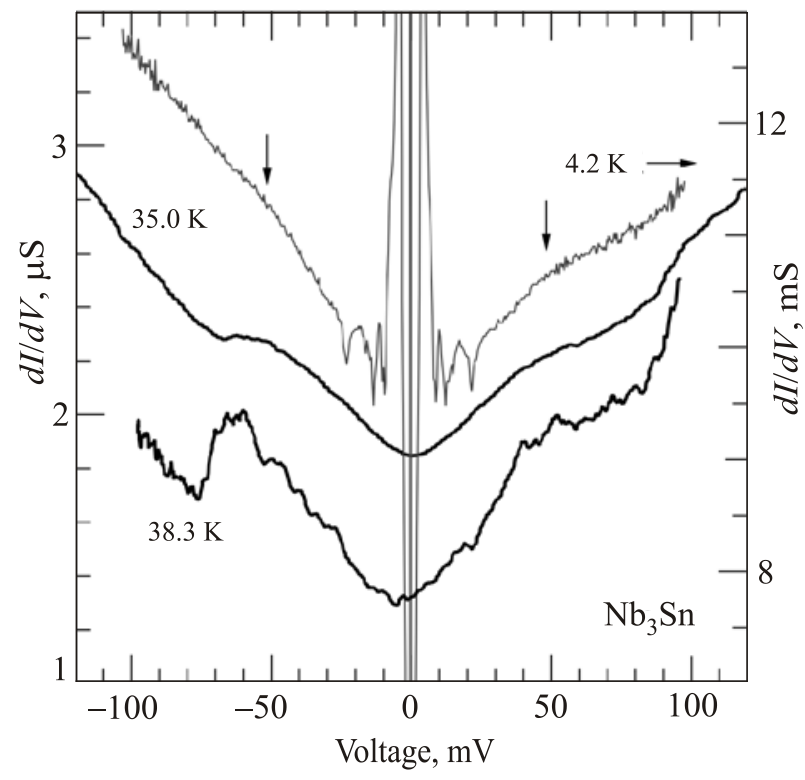

Fig. 4. $G(V)$ for different $\mathrm{Nb}_{3} \mathrm{Sn}$ break junctions in the superconducting and normal states, displaying the bias range up to $\pm 120 \mathrm{mV}$.

at $V=0$ and the hump structures at $\approx \pm 20 \mathrm{mV}$. Other peculiar features of $G(V)$ are the kink structures of $G(V)$ centered at biases $\approx \pm 50 \mathrm{mV}$ with a weak $(\approx 5 \%)$ excess over the background $G(V)$ level. At $T \approx 35-38 \mathrm{~K}$, i.e., well above $T_{C}$, the characteristic reproducible gap-edge structures are also observed at about \pm (50-60) $\mathrm{mV}$, which agrees with the kink structures of $\pm 50 \mathrm{mV}$ shown in $G(V)$ at $4.2 \mathrm{~K}$. It means that the energy gap of some nature appears in the normal state of $\mathrm{Nb}_{3} \mathrm{Sn}$. Note, that the normal-state gap edge positions $\approx \pm 50$ $\mathrm{mV}$ are roughly twice as large as those with $\pm(20-30) \mathrm{mV}$ found in the superconducting state and indicated in Fig. 4 as well as in Figs. 1 and 3. In particular, the above mentioned double-gap features inherent to the $23.9 \mathrm{~K}$ curve shown in Fig. 3 (at $\pm 20 \mathrm{mV}$ and $\pm 40 \mathrm{mV}$ ) can be attributed to the emergent symmetric SIS and nonsymmetric SIN junctions, respectively. The substantially suppressed sub-gap conductance features in the normal state $G(V)$ indicate that a certain quality of the junction interface should be realized to notice the partial density of states gapping. It is remarkable that the positions of the observed normal-state gap peculiarities at $\approx \pm(20-30) \mathrm{mV}$ and $\approx \pm(50-60) \mathrm{mV}$ do not change although the temperature was raised up to $\approx 40 \mathrm{~K}$.

The asymmetric character of $G(V)$ above $T_{C}$ in Fig. 3 can be understood when the phase of the order parameter of the CDW states is taken into account. This phase substantially distorts the quasiparticle tunneling characteristics in the partially CDW-gapped and partially normal-metal state formed in the symmetric set-up of the BJ between CDW metals. Hence, if the junction remains symmetric, the non-equality of $G(V)$ branches may be a consequence of the broken symmetry phenomenon $[15,16]$, when both of the electrodes are in the same CDW-gapped state but the order parameters exhibit different signs. In an alternative scenario, this asym- metry, together with the double-gap features at about inner values of $\pm 20 \mathrm{mV}$ and almost twice larger values $\pm 40 \mathrm{mV}$ formed at $23.9 \mathrm{~K}$, indicates that the gap structures possessing the values $\pm 20 \mathrm{mV}$ are most probably a consequence of the asymmetric junction formation with the CDW gap $\Sigma$ and a singularity at $V= \pm \Sigma /$ e. It might happen that an asymmetric junction is formed after cracking the sample with one of the electrodes being in a CDW-free metallic state. Then $G(V)$ is asymmetric $[15,16]$.

The existence of the CDW gap in the tunnel spectra of $\mathrm{Nb}_{3} \mathrm{Sn}$ impedes the conventional studies of the electronphonon interaction as strong-coupling features, which can be performed in more conventional superconductors [17]. Once such structures are found as the intrinsic gaps, like in our case, the difficulties of interpretation should be resolved taking into account the gapping by instabilities in the electron-hole channel. The weak hump structure depicted in Figs. 1 and 3 is now understood as the $\pm \Sigma / e$ singularity emerging due to the partial CDW gapping. The asymmetric form may be considered as the broken symmetry of CDW with the opposite signs of the order parameters. On the other hand, as we have indicated above, the asymmetric $G(V)$ shape for the apparently symmetric junction may arise due to the actual formation of the asymmetric (one-side-normal metallic) junction. In this case the CDW-driven current-voltage characteristics are asymmetric for any phase of $\Sigma$ except $\pi / 2[15,16]$. The realization of the actually asymmetric configuration in the nominally symmetric junctions was observed in the BJ measurements of both CDW conductors [18] and superconductors [19].

There is enough evidence to explain the emergence of the gap feature, which is due to the martensitic structural phase transition occurring in the A-15 compound like $\mathrm{Nb}_{3} \mathrm{Sn}[1,4]$. Below the corresponding transition temperature, the cubic crystallographic symmetry is violated and the tetragonal periodic lattice distortions appear driven by the Peierls-type instability due to the displacement of $\mathrm{Nb}$ atoms. The concomitant CDW leads to the quasiparticle gap formation in the parent electronic density of states $N(E)$. Since its electronic signature was believed to be very weak, there were previously not so many observations and discussions concerning the peculiarities of the tunnel conductance spectra of $\mathrm{Nb}_{3} \mathrm{Sn}$ junctions. Moreover, CDW distortions in $\mathrm{Nb}_{3} \mathrm{Sn}$ seem to be spatially inhomogeneous, which is similar to what is intrinsic to cuprate layered structures $[16,20]$. This would obscure the CDW electronic singularity as compared with the conventional sharp second-kind phase transitions. Therefore, the CDW gapping reveals itself in the tunneling $G(V)$ as a weak pseudogap feature [16,20-22].

The high- $T$ measurements clarified that the gap-edge value does not decrease as compared with the low temperature data even near $40 \mathrm{~K}$. Generally speaking, the inhomogeneity of the CDW formation should result in the crystallographic scattered CDW values. The broad peak 
structures at $\pm(50-60) \mathrm{mV}$ can be attributed to the scattered CDW gap edges with $\pm 2 \Sigma / e$ corresponding to the martensitic transition, which normally occurs at $T_{m}=$ $=T_{C D W}[1,23]$. According to our data, $T_{C D W}$ is assumed to be in the range $\approx 43 \mathrm{~K}$, so that the gap ratio $2 \Sigma / k_{B} T_{C D W}$ can be estimated as $\approx 14 \pm 2$. Such values are typical for CDW phase transitions. For instance, $2 \Sigma / k_{B} T_{C D W}$ is about 15 for the low-dimensional CDW conductor $\mathrm{NbSe}_{3}$ [24].

Finally, the theoretical approach to the interplay between superconductivity and CDW phenomena, which started in connection to A-15 compounds [4], was recently successfully applied to treat the pseudogap phenomena in copper oxides [15,16,20,21]. The presented studies of $\mathrm{Nb}_{3} \mathrm{Sn}$ strongly support the idea that the dip-hump structures in A-15 and high- $T_{C}$ superconductors are of a similar origin.

\section{Conclusions}

We have measured the $\mathrm{Nb}_{3} \mathrm{Sn}$ single crystal by the break junction tunneling spectroscopy. The maximum superconducting gap was found to be $2 \Delta \approx 5.5 \mathrm{meV}$, which corresponds to the gap ratio $2 \Delta / k_{B} T_{C} \approx 3.6-3.7$. We never observed the strong-coupling ratio $4.3-4.4$ reported elsewhere in the literature. The normal-state tunneling conductance exhibits the gap-like structure of $2 \Sigma=50-60 \mathrm{meV}$ at least up to $\approx 40 \mathrm{~K}$, which can be attributed to the CDW gap appearing due to the martensitic structural phase transition below $T_{m}$. The gap ratio $2 \Sigma / k_{B} T_{m}=13-16$ if one assumes $T_{m} \approx 43 \mathrm{~K}$ agrees with that found in the known CDW conductors.

\section{Acknowledgements}

This work was supported by a Grand-in-Aid for Scientific Research (245403770) of the Japan Society for the Promotion of Science (JSPS). The work was partially supported by the Project No. 8 of the 2012-2014 Scientific Cooperation Agreement between Poland and Ukraine.

1. L.R. Testardi, Rev. Mod. Phys. 47, 637 (1975).

2. F.E. Wang, J. Phys. Chem. Solids 35, 273 (1974).

3. L.P. Gorkov, Zh. Eksp. Teor. Fiz. 17, 525 (1973) [Sov. Phys. JETP 17, 830 (1974)].
4. G. Bilbro and W.L. McMillan, Phys. Rev. B 14, 1887 (1976).

5. E.L. Wolf, J. Zasadzinski, G.B. Arnold, D.F. More, J.M. Rowell, and M.R. Beasley, Phys. Rev. B 22, 1214 (1980).

6. D.A. Rudman, J. Appl. Phys. 55, 3544 (1984).

7. J. Geerk, U. Kaufmann, W. Bangert, and H. Rietschel, Phys. Rev. B 33, 1621 (1986).

8. J. Moreland and J.W. Ekin, J. Appl. Phys. 58, 3888 (1985).

9. R. Escudero and F. Morales, Solid State Commun. 150, 715 (2010).

10. T. Ekino, T. Takabatake, H. Tanaka, and H. Fujii, Phys. Rev. Lett. 75, 4262 (1995).

11. R.C. Dynes, V. Narayanamurti, and J.P. Garno, Phys. Rev. Lett. 41, 1509 (1978).

12. T. Ekino, A.M. Gabovich, and A.I. Voitenko. Fiz. Nizk. Temp. 34, 515 (2008) [Low Temp. Phys. 34, 409 (2008)].

13. L.Y.L. Shen, Phys. Rev. Lett. 29, 1082 (1972).

14. S.I. Vedeneev, A.I. Golovashkin, I.S. Levchenko, and G.P. Motulevich, Zh. Eksp. Teor. Fiz. 63, 1010 (1972) [Sov. Phys. JETP 36, 531 (1973)].

15. A.M. Gabovich, A.I. Voitenko, and M. Ausloos, Phys. Rep. 367, 583 (2002).

16. A.M. Gabovich, A.I. Voitenko, T. Ekino, M.S. Li, H. Szymczak, and M. Pękała, Adv. Condens. Matter Phys. 2010, 681070 (2010).

17. J. Geerk and H.v. Löhneysen, Phys. Rev. Lett. 99, 257005 (2007).

18. M.H. Jung, T. Ekino, Y.S. Kwon, and T. Takabatake, Phys. Rev. B 63, 035101 (2001).

19. T. Ekino, H. Fujii, M. Kosugi, Y. Zenitani, and J. Akimitsu, Phys. Rev. B 53, 5640 (1996).

20. T. Ekino, A.M. Gabovich, M.S. Li, M. Pękała, H. Szymczak, and A.I. Voitenko, Phys. Rev. B 76, 180503 (2007).

21. T. Ekino, A.M. Gabovich, M.S. Li, M. Pękała, H. Szymczak, and A.I. Voitenko, J. Phys.: Condens. Matter 23, 385701 (2011).

22. P. Monceau, Adv. Phys. 61, 325 (2012).

23. J. Müller, Rep. Prog. Phys. 43, 643 (1980).

24. T. Ekino and J. Akimitsu, Jpn. J. Appl. Phys. Suppl. 26, 625 (1987). 